\title{
On the Largest Disc Mapped by Sum of Convex and Starlike Functions
}

\author{
Rosihan M. Ali, ${ }^{1}$ Naveen Kumar Jain, ${ }^{2}$ and V. Ravichandran ${ }^{2}$ \\ ${ }^{1}$ School of Mathematical Sciences, Universiti Sains Malaysia (USM), 11800 Penang, Malaysia \\ ${ }^{2}$ Department of Mathematics, University of Delhi, Delhi 110007, India
}

Correspondence should be addressed to Rosihan M. Ali; rosihan@cs.usm.my

Received 5 July 2013; Accepted 17 October 2013

Academic Editor: Ferhan M. Atici

Copyright (C) 2013 Rosihan M. Ali et al. This is an open access article distributed under the Creative Commons Attribution License, which permits unrestricted use, distribution, and reproduction in any medium, provided the original work is properly cited.

For a normalized analytic function $f$ defined on the unit disc $\mathbb{D}$, let $\phi\left(f, f^{\prime}, f^{\prime \prime} ; z\right)$ be a function of positive real part in $\mathbb{D}$, $\psi\left(f, f^{\prime}, f^{\prime \prime} ; z\right)$ need not have that property in $\mathbb{D}$, and $\chi=\phi+\psi$. For certain choices of $\phi$ and $\psi$, a sharp radius constant $\rho$ is determined, $0<\rho<1$, so that $\chi(\rho z) / \rho$ maps $\mathbb{D}$ onto a specified region in the right half-plane.

\section{Introduction}

Let $\mathscr{A}$ be the class of functions $f$ analytic in $\mathbb{D}=\{z \in$ $\mathbb{C}:|z|<1\}$ and normalized by $f(0)=0=f^{\prime}(0)-1$. Let $\mathcal{S}$ be its subclass consisting of univalent functions. For two analytic functions $f$ and $g$, the function $f$ is subordinate to $g$, written $f(z) \prec g(z)$, if there is an analytic selfmap $w: \mathbb{D} \rightarrow \mathbb{D}$ with $w(0)=0$ satisfying $f(z)=g(w(z))$. Given an analytic function $p$ with $p(0)=1$ and Re $p(z)>$ 0 in $\mathbb{D}$, denote by $\mathcal{S} \mathscr{T}(p)$ and $\mathscr{C} \mathscr{V}(p)$ the subclasses of $\mathscr{A}$ consisting, respectively, of $f$ satisfying $z f^{\prime}(z) / f(z) \prec$ $p(z)$ and $1+z f^{\prime \prime}(z) / f^{\prime}(z)<p(z)$.

For various choices of $p$, these classes reduce to wellknown subclasses of starlike and convex functions. For instance, with $p(z)=(1+(1-2 \alpha) z) /(1-z), 0 \leq \alpha<$ 1 , then $\mathscr{S} \mathscr{T}(\alpha)$ and $\mathscr{C} \mathscr{V}(\alpha)$ are, respectively, the subclasses consisting of starlike functions of order $\alpha$ and convex functions of order $\alpha$. The classes $\mathcal{S} \mathscr{T}:=\mathcal{S} \mathscr{T}(0)$ and $\mathscr{C} \mathscr{V}:=$ $\mathscr{C} \mathscr{V}(0)$ are the familiar subclasses of $\delta$ of starlike and convex functions. For $p(z)=(1+(1-2 \beta) z) /(1-z), \beta>1$, $\mathscr{M}(\beta)=\mathcal{S} \mathscr{T}(p)$ is the class of functions $f \in \mathscr{A}$ satisfying

$$
\mathscr{M}(\beta):=\left\{f \in \mathscr{A}: \operatorname{Re}\left(\frac{z f^{\prime}(z)}{f(z)}\right)<\beta(z \in \mathbb{D})\right\}
$$

studied by Uralegaddi et al. [1]. Various subclasses of $\mathscr{M}(\beta)$ have been investigated in [2-5]. For $p(z)=$ $((1+z) /(1-z))^{\alpha}, 0<\gamma \leq 1$, the class $\mathcal{S} \mathcal{S} \mathscr{T}(\gamma):=\mathcal{S} \mathscr{T}(p)$ is the class of strongly starlike functions of order $\gamma$. The class $\mathcal{S}_{\mathscr{L}}:=\quad \mathcal{S} \mathscr{T}(\sqrt{1+z})$ introduced by Sokół and Stankiewicz [6] consists of functions $f \in \mathscr{A}$ satisfying

$$
\left|\left(\frac{z f^{\prime}(z)}{f(z)}\right)^{2}-1\right|<1 \quad(z \in \mathbb{D}) .
$$

Thus, a function $f \in \mathscr{A}$ is in the class $\delta_{\mathscr{L}}$ if $z f^{\prime}(z) / f(z)$ lies in the region bounded by the right-half of the lemniscate of Bernoulli given by $\left|w^{2}-1\right|<1$. Results related to the class $\delta_{\mathscr{L}}$ can be found in $[3,7-9]$.

In investigating the class $\mathscr{U} \mathscr{C} \mathscr{V}$ of uniformly convex functions, Rønning [10] introduced a class $\delta_{\mathscr{P}}$ of parabolic starlike functions. These are functions $f \in \mathscr{A}$ satisfying

$$
\operatorname{Re}\left(\frac{z f^{\prime}(z)}{f(z)}\right)>\left|\frac{z f^{\prime}(z)}{f(z)}-1\right| \quad(z \in \mathbb{D})
$$

It is important to keep in mind that the qualifier "parabolic" refers to the geometry of the image of $\mathbb{D}$ under the map $z f^{\prime}(z) / f(z)$; that is, the domain necessarily lies in a parabolic region of the $w$-plane. It does not convey the interpretation that the function $f$ maps the disk $\mathbb{D}$ onto a parabolic region. This terminology of parabolic starlike functions is however widely accepted and used by authors. Ali 
and Ravichandran [11] recently surveyed works on uniformly convex and parabolic starlike functions.

This paper finds radius estimates for classes of functions in $\mathscr{A}$. The radius of a property $P$ in a given set of functions $\mathscr{M}[12$, page 119] is the largest number $R$ such that every function in the set $\mathscr{M}$ has the property $P$ in each disc $\mathbb{D}_{r}=\{z \in \mathbb{C}:|z|<r\}$ for every $r<R$. For example, the Koebe function $k(z)=z /(1-z)^{2}$, which maps $\mathbb{D}$ onto the domain $\mathbb{C} \backslash\{w \in \mathbb{R}: w \leq-1 / 4\}$, is starlike but not convex. However, $k$ maps the disc $\mathbb{D}_{r}$ onto a convex domain for every $r \leq 2-\sqrt{3}$. Indeed, every univalent function $f \in$ $\mathcal{S}$ maps $\mathbb{D}_{r}$ onto a convex domain for $r \leq 2-\sqrt{3}[13$, Theorem 2.13, page 44]. This number is known as the radius of convexity for $\mathcal{S}$.

It is known that $\mathscr{C} \mathscr{V} \subseteq \mathcal{S T}(1 / 2) \subseteq\{f \in \mathscr{A}$ : $\operatorname{Re}(f(z) / z)>1 / 2, z \in \mathbb{D}\}$. The function $g(z)=z /(1-z)$ is convex and therefore starlike of order $1 / 2$; it is clear that the function

$$
\phi(z):=\frac{z g^{\prime}(z)}{g(z)}
$$

has real part greater than $1 / 2$. Now the function

$$
\psi(z):=\frac{z^{2} g^{\prime \prime}(z)}{g(z)}
$$

takes values in $\mathbb{C} \backslash\{w \in \mathbb{R}: w \leq-1 / 2\}$, and therefore does not have positive real part for all $z \in \mathbb{D}$. Their sum

$$
\begin{aligned}
\phi(z)+\psi(z) & =\frac{z g^{\prime}(z)}{g(z)}+\frac{z^{2} g^{\prime \prime}(z)}{g(z)} \\
& =\frac{z g^{\prime}(z)}{g(z)}\left(1+\frac{z g^{\prime \prime}(z)}{g^{\prime}(z)}\right)
\end{aligned}
$$

takes values in $\left\{w:=x+i y \in \mathbb{C}: y^{2}>-x / 2\right\}$ and therefore the sum $\phi+\psi$ does not have positive real part in $\mathbb{D}$. This motivates us to determine the largest radius $\rho$ such that

$$
\operatorname{Re}\left(\frac{z^{2} g^{\prime \prime}(z)}{g(z)}+\frac{z g^{\prime}(z)}{g(z)}\right)>\alpha \quad(|z| \leq \rho) .
$$

More generally, let $\phi=\phi\left(f, f^{\prime}, f^{\prime \prime} ; z\right)$ and $\psi=\psi\left(f, f^{\prime}\right.$, $\left.f^{\prime \prime} ; z\right)$ be functions satisfying $\operatorname{Re} \phi>0$ in $\mathbb{D}$, while $\operatorname{Re} \psi$ need not necessarily be positive in the whole unit disc $\mathbb{D}$. For certain choices of $\phi$ and $\psi$, a sharp radius constant $\rho$ is determined, $0 \leq \rho<1$, so that whenever $|z|<\rho$, the sum $\phi+\psi$ takes values in specified regions in the complex plane. The results obtained are shown to reduce those of Singh and Paul [14] in certain special cases.

\section{Main Results}

For $\phi(z):=\phi\left(f, f^{\prime}, f^{\prime \prime} ; z\right)=z f^{\prime}(z) / f(z)$ and $\psi(z):=$ $\psi\left(f, f^{\prime}, f^{\prime \prime} ; z\right)=z^{2} f^{\prime \prime}(z) / f(z)$, with $f \in \mathcal{S} \mathscr{T}(1 / 2)$, several radius results for the sum $\phi+\psi$ to be in certain regions in the complex plane are obtained in the following result.
Theorem 1. Let $f \in \mathcal{S} \mathscr{T}(1 / 2)$; let $\chi: \mathbb{D} \rightarrow \mathbb{C}$ be defined by

$$
\begin{gathered}
\chi(z)=\frac{z^{2} f^{\prime \prime}(z)}{f(z)}+\frac{z f^{\prime}(z)}{f(z)}, \\
\chi_{i}(z)=\chi\left(\rho_{i} z\right), \quad i=1,2, \ldots, 6 .
\end{gathered}
$$

Then

(a) $\operatorname{Re} \chi_{1}(z)>\alpha, 0 \leq \alpha<1$, where $\rho_{1}$ is given by

$\rho_{1}= \begin{cases}\sqrt{\frac{7-16 \alpha}{11-16 \alpha+8 \sqrt{2-4 \alpha}},}, & 0 \leq \alpha \leq \frac{2+\sqrt{13}}{18} \\ \frac{2(1-\alpha)}{1+2 \alpha+\sqrt{1+8 \alpha}}, & \frac{2+\sqrt{13}}{18}<\alpha<1 .\end{cases}$

(b) $\left|\chi_{2}^{2}(z)-1\right|<1$, where $\rho_{2}$ is given by

$$
\rho_{2}=\frac{2(\sqrt{2}-1)}{1+2 \sqrt{2}+\sqrt{1+8 \sqrt{2}}} \simeq 0.112903 .
$$

(c) $\operatorname{Re} \chi_{3}(z)<\beta, \beta>1$, where $\rho_{3}$ is given by

$$
\rho_{3}=\frac{2(\beta-1)}{1+2 \beta+\sqrt{1+8 \beta}} .
$$

(d) $\left|\chi_{4}(z)-1\right|<1-\alpha, 0 \leq \alpha<1$, where $\rho_{4}$ is given by

$$
\rho_{4}=\frac{2(1-\alpha)}{5-2 \alpha+\sqrt{17-8 \alpha}} .
$$

(e) $\left|\arg \left(\chi_{5}(z)\right)\right|<\gamma \pi / 2,0<\gamma \leq 1$ where $\rho_{5}=\rho_{5}(\gamma) \epsilon$ $(0,1)$ is the root of the equation in $r$ :

$$
\begin{gathered}
\left(1+2\left(1-r^{2}\right) t_{0}\right) \sqrt{4 t_{0}-\left(1+t_{0}-r^{2} t_{0}\right)^{2}} \\
-\left(1+t_{0}\left(-1-3 r^{2}+2\left(1-r^{2}\right)^{2} t_{0}\right)\right) \tan \left(\frac{\pi \gamma}{2}\right)=0, \\
t_{0}=\frac{5-r^{2}+\sqrt{9-10 r^{2}+17 r^{4}}}{8\left(1-r^{4}\right)} .
\end{gathered}
$$

In particular,

$$
\begin{aligned}
\rho_{5}\left(\frac{1}{4}\right) & \simeq 0.131522, & \rho_{5}\left(\frac{1}{2}\right) & \simeq 0.266747 \\
\rho_{5}\left(\frac{3}{4}\right) & \simeq 0.409049, & \rho_{5}(1) & \simeq 0.560097 .
\end{aligned}
$$

(f) Also $\left|\chi_{6}(z)-1\right|<\operatorname{Re} \chi_{6}(z)$, where $\rho_{6} \simeq 0.23605 \epsilon$ $(0,1)$ is the root of the following equation in $r$ :

$$
\begin{aligned}
& 2\left(1+9 r^{2}\right) t_{0}-1+\left(-5+26 r^{2}-21 r^{4}\right) t_{0}^{2} \\
& \quad+4\left(1-r^{2}\right)^{2}\left(1+3 r^{2}\right) t_{0}^{3}-4\left(1-r^{2}\right)^{4} t_{0}^{4}=0,
\end{aligned}
$$


and $t_{0} \in\left(1 /(1+r)^{2}, 1 /(1-r)^{2}\right)$ is the root of the equation in $t$ :

$$
\begin{aligned}
& 2\left(1+9 r^{2}\right)+2\left(-5+26 r^{2}-21 r^{4}\right) t \\
& \quad+12\left(1-r^{2}\right)^{2}\left(1+3 r^{2}\right) t^{2}-16\left(1-r^{2}\right)^{4} t^{3}=0 .
\end{aligned}
$$

Each radius constant $\rho_{i}$ is sharp.

For two analytic functions $f, g \in \mathscr{A}$, their convolution or Hadamard product, denoted by $f * g$, is defined by $(f *$ $g)(z):=z+\sum_{n=2}^{\infty} a_{n} b_{n} z^{n}$. The following results are needed in the sequel.

Lemma 2 ([15, Lemma 2.7, page 126; Lemma 3.5, page 130]). If $f \in \mathscr{C} \mathscr{V}$ and $g \in \mathcal{S} \mathscr{T}$, or $f$ and $g$ belong to $\mathcal{S} \mathscr{T}(1 / 2)$, then

$$
\frac{f * g F}{f * g}(\mathbb{D}) \subset \overline{\mathrm{CO}}(F(\mathbb{D}))
$$

for any function $F$ analytic in $\mathbb{D}$, where $\overline{\mathrm{co}}(F(\mathbb{D}))$ denotes the closed convex hull of $F(\mathbb{D})$.

Lemma 3 ([7, Lemma 2.2, page 6559]). For $0<a<\sqrt{2}$, let $r_{a}$ be given by

$$
r_{a}= \begin{cases}\left(\sqrt{1-a^{2}}-\left(1-a^{2}\right)\right)^{1 / 2}, & 0<a \leq 2 \sqrt{2} / 3 \\ \sqrt{2}-a, & 2 \sqrt{2} / 3 \leq a<\sqrt{2},\end{cases}
$$

and for $a>0$, let $R_{a}$ be given by

$$
R_{a}= \begin{cases}\sqrt{2}-a, & 0<a \leq \frac{1}{\sqrt{2}} \\ a, & \frac{1}{\sqrt{2}} \leq a .\end{cases}
$$

Then,

$$
\begin{aligned}
\left\{w:|w-a|<r_{a}\right\} & \subseteq\left\{w:\left|w^{2}-1\right|<1\right\} \\
& \subseteq\left\{w:|w-a|<R_{a}\right\} .
\end{aligned}
$$

Proof of Theorem 1. Let $h: \mathbb{D} \rightarrow \mathbb{C}$ be defined by

$$
h(z)=\frac{2}{(1-z)^{2}}-\frac{1}{1-z} .
$$

First, for each $i=1,2, \ldots, 6, h_{i}(z)=h\left(\rho_{i} z\right)$ will be shown to, respectively, satisfy $\operatorname{Re} h_{1}(z)>\alpha,\left|h_{2}(z)-1\right|<$ $1, \operatorname{Re} h_{3}(z)<\beta,\left|h_{4}(z)-1\right|<1-\alpha,\left|\arg h_{5}(z)\right|<\gamma \pi / 2$, and $\left|h_{6}(z)-1\right|<\operatorname{Re} h_{6}(z)$. Then, using Lemma 2 , $\chi_{i}$ is deduced to satisfy the required condition.

As in [14], let

$$
\frac{1}{1-z}=R e^{i \theta}
$$

so that

$$
\begin{gathered}
\frac{1}{1+r} \leq R \leq \frac{1}{1-r} \quad(|z|=r), \\
\cos \theta=\frac{1+R^{2}-r^{2} R^{2}}{2 R} .
\end{gathered}
$$

(a) By (21), (22), and (23), it follows that

$$
\begin{aligned}
\operatorname{Re} h(z) & =2 R^{2} \cos 2 \theta-R \cos \theta \\
& =\frac{1}{2}-\frac{1}{2}\left(1+3 r^{2}\right) t+\left(1-r^{2}\right)^{2} t^{2} \quad\left(t:=R^{2}\right) \\
& =: \phi(t) .
\end{aligned}
$$

Case (i). Suppose that $0 \leq \alpha \leq(2+\sqrt{13}) / 18$. We assert that $\min \phi(t)>\alpha$ for $|z|<\rho_{1}$, where the minimum is taken over all $t \in\left(1 /(1+r)^{2}, 1 /(1-r)^{2}\right)$. Let $r<\rho_{1}$. Then

$$
\frac{\partial \phi(t)}{\partial t}=-\frac{1}{2}\left(1+3 r^{2}\right)+2\left(1-r^{2}\right)^{2} t=0
$$

if $t=t_{0}:=\left(1+3 r^{2}\right) /\left(4\left(1-r^{2}\right)^{2}\right), \partial^{2} \phi\left(t_{0}\right) / \partial t^{2}>0$, and that for $r \geq 4-\sqrt{13}$,

$$
\frac{1}{(1+r)^{2}} \leq t_{0} \leq \frac{1}{(1-r)^{2}} .
$$

Thus, for $4-\sqrt{13} \leq r<\rho_{1}$,

$$
\min \phi(t)=\phi\left(t_{0}\right)=\frac{7-22 r^{2}-r^{4}}{16\left(1-r^{2}\right)^{2}}>\alpha .
$$

On the other hand, if $r<4-\sqrt{13}$, then it can be shown that

$$
\begin{aligned}
\min \phi(t) & =\phi\left(\frac{1}{(1+r)^{2}}\right) \\
& =\frac{1-r}{(1+r)^{2}}>\frac{\sqrt{13}-3}{(5-\sqrt{13})^{2}} \\
& =\frac{2+\sqrt{13}}{18}>\alpha
\end{aligned}
$$

Case (ii). For $(2+\sqrt{13}) / 18<\alpha<1$, then $\min \phi(t)>$ $\alpha$ in $|z|<\rho_{1}, t \in\left(1 /(1+r)^{2}, 1 /(1-r)^{2}\right)$. Indeed for $r<$ $\rho_{1}<4-\sqrt{13}$, as in the case (i),

$$
\min \phi(t)=\phi\left(\frac{1}{(1+r)^{2}}\right)=\frac{1-r}{(1+r)^{2}}>\alpha .
$$

The previously mentioned two cases show that $\operatorname{Re} h_{1}(z)>$ $\alpha$ in $\mathbb{D}$. Figure 1 illustrates sharpness of the radius $\rho_{1}=\sqrt{5}-$ 2 in the case $\alpha=0.5$.

(b) For $h$ given by (21), a calculation shows that

$$
\begin{aligned}
|h(z)-1| & =\left|\frac{2}{(1-z)^{2}}-\frac{1}{1-z}-1\right| \\
& =\left|\frac{z}{1-z}+\frac{2 z}{(1-z)^{2}}\right| \\
& \leq \frac{r}{1-r}+\frac{2 r}{(1-r)^{2}}
\end{aligned}
$$




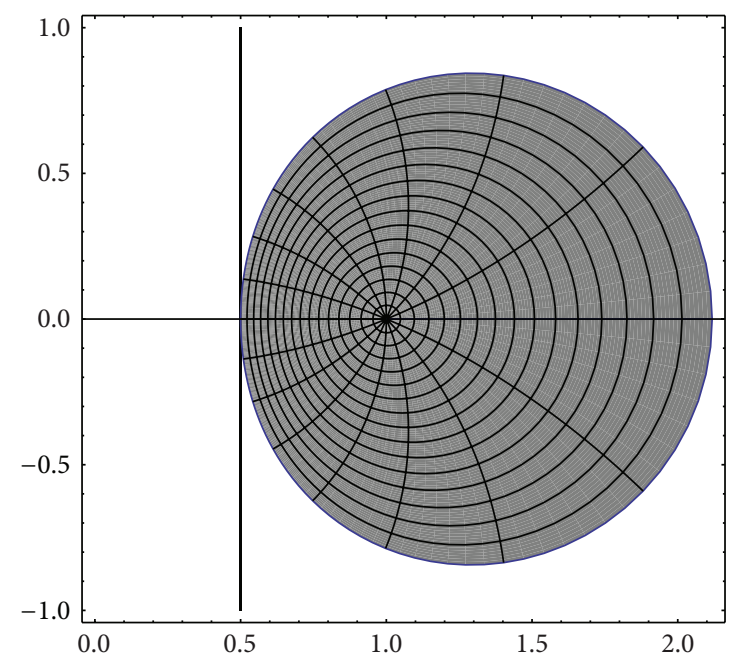

FIgURE 1: Image of $|z| \leq \sqrt{5}-2$ touches Re $w=0.5$.

By Lemma 3, the function $h$ satisfies

$$
\left|h^{2}(z)-1\right|<1
$$

provided

$$
\frac{r}{1-r}+\frac{2 r}{(1-r)^{2}} \leq \sqrt{2}-1
$$

that is,

$$
\sqrt{2} r^{2}-(1+2 \sqrt{2}) r+(\sqrt{2}-1) \geq 0 \text {. }
$$

This inequality holds if $r \leq \rho_{2}$. Figure 2 illustrates sharpness of the radius $\rho_{2} \simeq 0.1129$.

(c) From (30), it follows that

$$
\operatorname{Re} h(z) \leq 1+\frac{r}{1-r}+\frac{2 r}{(1-r)^{2}} \leq \beta
$$

provided

$$
\beta r^{2}-(1+2 \beta) r-(1-\beta) \geq 0
$$

holds, which occurs whenever $r \leq \rho_{3}$. Sharpness of the radius $\rho_{3}=(4-\sqrt{13}) / 3$ in the case $\beta=1.5$ is illustrated in Figure 3.

(d) Inequality (30) also yields

$$
|h(z)-1| \leq \frac{r}{1-r}+\frac{2 r}{(1-r)^{2}} \leq 1-\alpha
$$

provided

$$
r^{2}(\alpha-2)+r(5-2 \alpha)+\alpha-1 \leq 0,
$$

that is, when $r \leq \rho_{4}$. Figure 4 illustrates sharpness of the radius $\rho_{4}=(4-\sqrt{13}) / 3$ in the case $\alpha=0.5$.

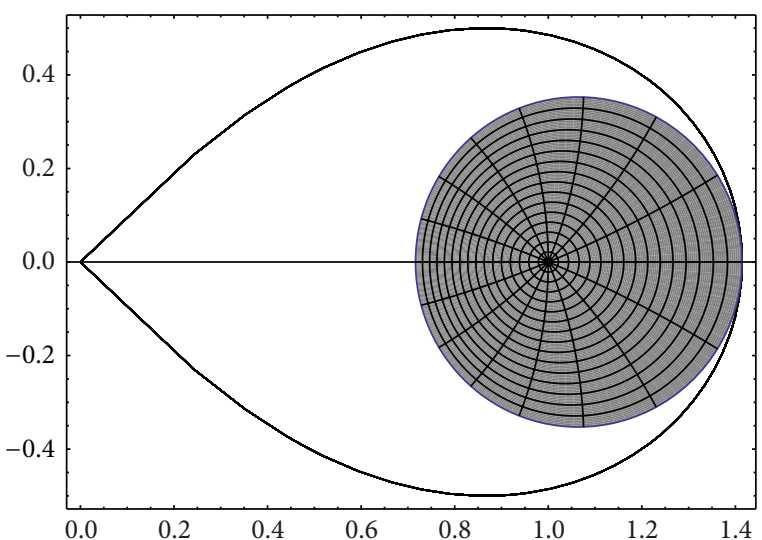

FigURE 2: Image of $|z| \leq 0.1129$ touches $\left|w^{2}-1\right|=1$.

(e) For the function $h$ given by (21), it follows from (22) and (23) that

$\arg h(z)$

$$
\begin{aligned}
& =\arctan \left(\frac{2 R \sin 2 \theta-\sin \theta}{2 R \cos 2 \theta-\cos \theta}\right) \\
& =\arctan \left(\frac{(4 R \cos \theta-1) \sin \theta}{2 R\left(2 \cos ^{2} \theta-1\right)-\cos \theta}\right) \\
& =\arctan \left(\frac{\left(1+2\left(1-r^{2}\right) R^{2}\right) \sqrt{4 R^{2}-\left(1+\left(1-r^{2}\right) R^{2}\right)^{2}}}{1-\left(1+3 r^{2}\right) R^{2}+2\left(1-r^{2}\right)^{2} R^{4}}\right) \\
& =\arctan \left(\frac{\left(1+2\left(1-r^{2}\right) t\right) \sqrt{4 t-\left(1+\left(1-r^{2}\right) t\right)^{2}}}{1-\left(1+3 r^{2}\right) t+2\left(1-r^{2}\right)^{2} t^{2}}\right) \\
& :=\arctan (\phi(t)),
\end{aligned}
$$

$t \in\left(1 /(1+r)^{2}, 1 /(1-r)^{2}\right)$. A calculation shows that $\phi^{\prime}(t)=$ 0 where

$$
\begin{aligned}
t= & t_{0} \\
& =\frac{5-r^{2}+\sqrt{9-10 r^{2}+17 r^{4}}}{8\left(1-r^{4}\right)} \in\left(\frac{1}{(1+r)^{2}}, \frac{1}{(1-r)^{2}}\right) .
\end{aligned}
$$

Now $\phi^{\prime}(t)>0$ for $t<t_{0}, \phi^{\prime}(t)<0$ if $t>t_{0}$, and

$$
\phi\left(\frac{1}{(1+r)^{2}}\right)=\phi\left(\frac{1}{(1-r)^{2}}\right)=0 \text {. }
$$

Thus

$\max \phi(t)=\phi\left(t_{0}\right)$

$$
=\frac{\left(1+2\left(1-r^{2}\right) t_{0}\right) \sqrt{4 t_{0}-\left(1+\left(1-r^{2}\right) t_{0}\right)^{2}}}{1-\left(1+3 r^{2}\right) t_{0}+2\left(1-r^{2}\right)^{2} t_{0}^{2}} .
$$




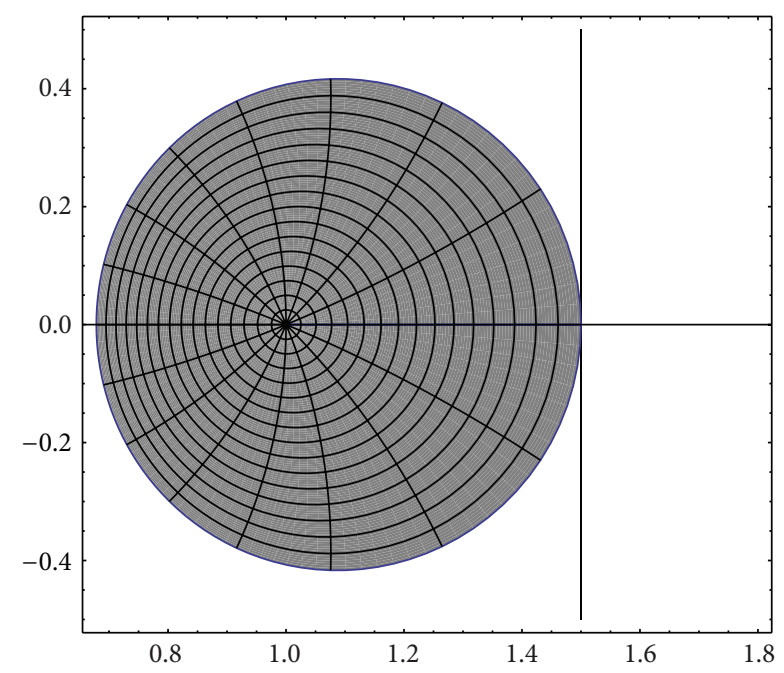

Figure 3: Image of $|z| \leq(4-\sqrt{13}) / 3$ touches Re $w=1.5$.

Evidently, (38) and (41) give

$|\arg h(z)|$

$$
\begin{aligned}
& \leq\left|\arctan \left(\frac{\left(1+2\left(1-r^{2}\right) t_{0}\right) \sqrt{4 t_{0}-\left(1+\left(1-r^{2}\right) t_{0}\right)^{2}}}{1-\left(1+3 r^{2}\right) t_{0}+2\left(1-r^{2}\right)^{2} t_{0}^{2}}\right)\right| \\
& \leq \frac{\gamma \pi}{2}
\end{aligned}
$$

provided

$$
\begin{aligned}
& \left(1+2\left(1-r^{2}\right) t_{0}\right) \sqrt{4 t_{0}-\left(1+\left(1-r^{2}\right) t_{0}\right)^{2}} \\
& \quad-\left(1+t_{0}\left(-1-3 r^{2}+2\left(1-r^{2}\right)^{2} t_{0}\right)\right) \tan \left(\frac{\pi \gamma}{2}\right) \\
& \leq 0 .
\end{aligned}
$$

Figure 5 illustrates sharpness of the radius $\rho_{5} \simeq$ 0.266747 in the case $\gamma=0.5$.

(f) The inequality

$$
|h(z)-1|<\operatorname{Re} h(z)
$$

holds if

$$
\begin{aligned}
& \left(2 R^{2} \cos 2 \theta-R \cos \theta-1\right)^{2}+\left(2 R^{2} \sin 2 \theta-R \sin \theta\right)^{2} \\
& \quad<\left(2 R^{2} \cos 2 \theta-R \cos \theta\right)^{2},
\end{aligned}
$$

or, with $t=R^{2}$,

$$
\begin{aligned}
\phi(t):= & 2\left(1+9 r^{2}\right) t-1+\left(-5+26 r^{2}-21 r^{4}\right) t^{2} \\
& +4\left(1-r^{2}\right)^{2}\left(1+3 r^{2}\right) t^{3}-4\left(1-r^{2}\right)^{4} t^{4}<0,
\end{aligned}
$$

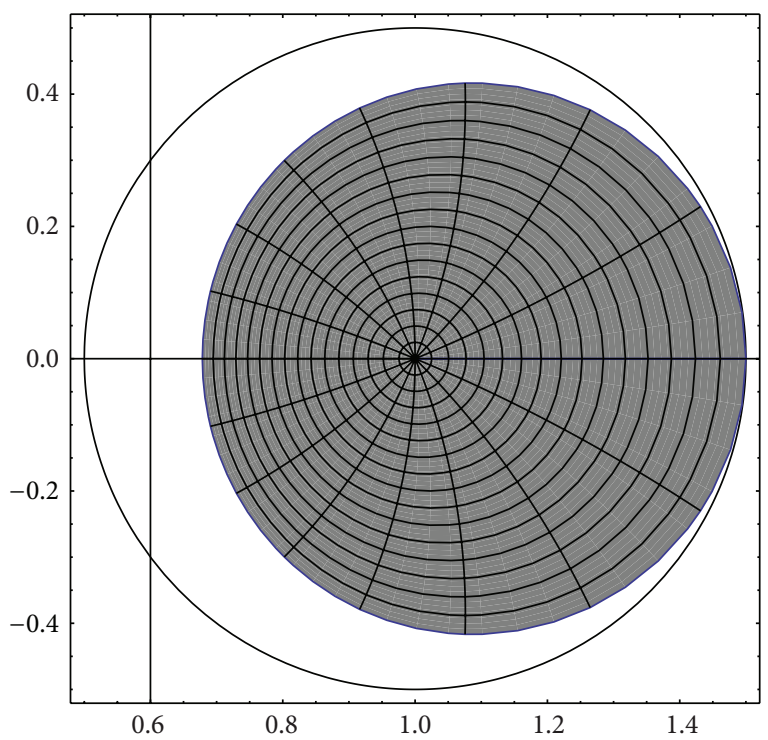

FIgURE 4: Image of $|z| \leq(4-\sqrt{13}) / 3$ touches $|w-1|=0.5$.

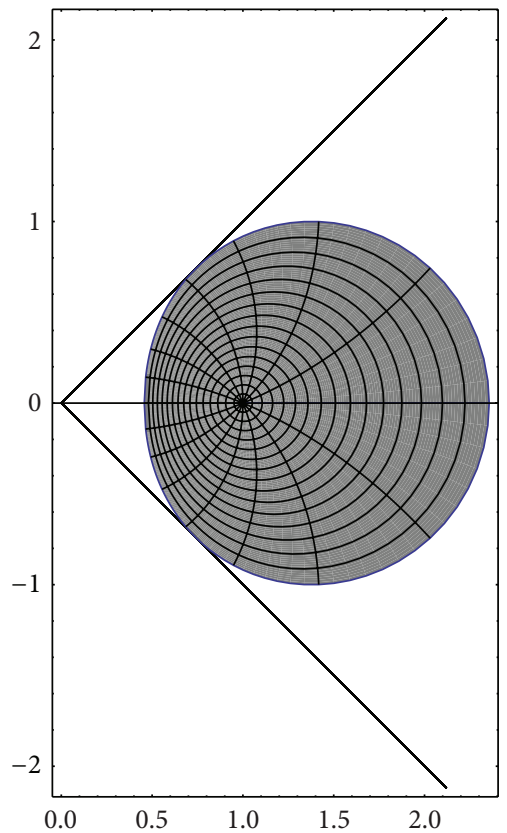

FIGURE 5: Image of $|z| \leq 0.266747$ touches $|\arg w|=\pi / 4$.

$$
\begin{aligned}
\phi^{\prime}(t)= & 2\left(1+9 r^{2}\right)+2\left(-5+26 r^{2}-21 r^{4}\right) t \\
& +12\left(1-r^{2}\right)^{2}\left(1+3 r^{2}\right) t^{2}-16\left(1-r^{2}\right)^{4} t^{3} .
\end{aligned}
$$




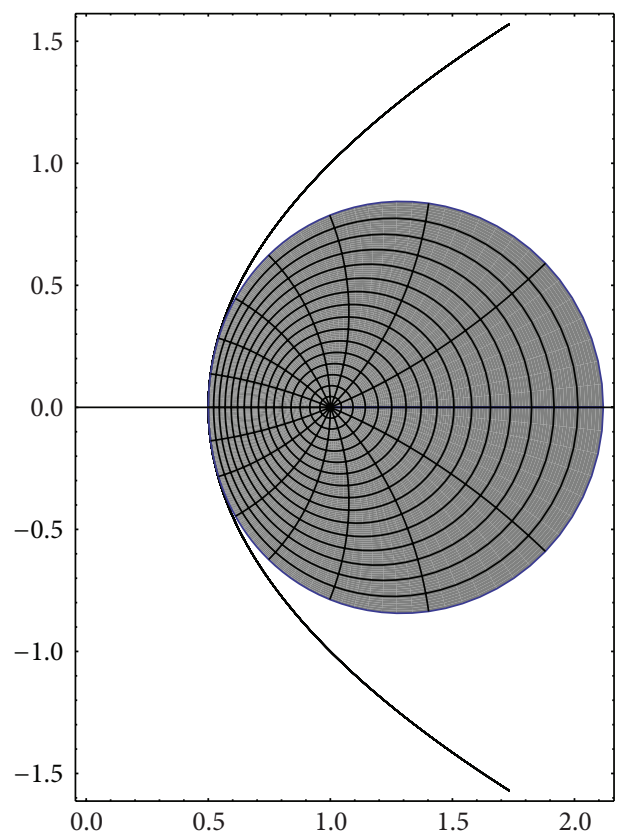

Figure 6: Image of $|z| \leq 0.23605$ touches $|w-1|=\operatorname{Re} w$.

Let $r<\rho_{6}$. Since

$$
\begin{gathered}
\phi^{\prime}\left(\frac{1}{(1+r)^{2}}\right) \\
=\frac{4\left(-3+11 r+6 r^{2}+7 r^{3}-r^{4}\right)}{(1+r)^{2}}>0 \quad \text { if } r>0.234722, \\
\phi^{\prime}\left(\frac{1}{(1-r)^{2}}\right)=-\frac{4\left(3+11 r-6 r^{2}+7 r^{3}+r^{4}\right)}{(1-r)^{2}}<0
\end{gathered}
$$

there exists a unique $t_{0} \in\left(1 /(1+r)^{2}, 1 /(1-r)^{2}\right)$ such that $\phi^{\prime}\left(t_{0}\right)=0$ and $\max \phi(t)=\phi\left(t_{0}\right)$.

Thus, $\max \phi(t)=\phi\left(t_{0}\right)<0$ for $0.234722<r<\rho_{6}$. When $r \leq 0.234722$,

$$
\max \phi(t)=\phi\left(\frac{1}{(1+r)^{2}}\right)=\frac{4\left(4 r-1+r^{2}\right)}{(1+r)^{2}}<0
$$

hence, $\phi(t)<0$ for $r<\rho_{6}$. Figure 6 illustrates sharpness of the radius $\rho_{6} \simeq 0.23605$.

Next, consider $g_{i}(z)=z /\left(1-\rho_{i} z\right) \in \mathscr{C} \mathscr{V} \subset \mathcal{S} \mathscr{T}(1 / 2), i=$ $1,2, \ldots, 6$. Then,

$$
\begin{aligned}
& \frac{f(z) * g_{i}(z) h_{i}(z)}{f(z) * g_{i}(z)} \\
& \quad=\frac{f(z) *\left(z /\left(1-\rho_{i} z\right)\right)\left(2 \rho_{i} z /\left(1-\rho_{i} z\right)^{2}+1 /\left(1-\rho_{i} z\right)\right)}{f(z) *\left(z /\left(1-\rho_{i} z\right)\right)}
\end{aligned}
$$

$$
\begin{aligned}
& =\frac{f(z) *\left(2 \rho_{i} z^{2} /\left(1-\rho_{i} z\right)^{3}+z /\left(1-\rho_{i} z\right)^{2}\right)}{f(z) *\left(z /\left(1-\rho_{i} z\right)\right)} \\
& =\frac{\rho_{i}^{2} z^{2} f^{\prime \prime}\left(\rho_{i} z\right)+\rho_{i} z f^{\prime}\left(\rho_{i} z\right)}{f\left(\rho_{i} z\right)} \\
& =\chi\left(\rho_{i} z\right)=\chi_{i}(z) .
\end{aligned}
$$

Lemma 2, together with (50) and the corresponding inequality for the function $h_{i}$, shows that each function $\chi_{i}$ satisfies the required condition. For sharpness, consider the function $f_{0}(z)=z /(1-z) \in \mathscr{C} \mathscr{V} \subset \mathcal{S} \mathscr{T}(1 / 2)$. Then,

$$
\frac{z^{2} f_{0}^{\prime \prime}(z)+z f_{0}^{\prime}(z)}{f_{0}(z)}=\frac{2}{(1-z)^{2}}-\frac{1}{1-z}=h(z) .
$$

Sharpness of the numbers $\rho_{i}$ is now evident in view of the definition $h$.

For $\alpha=0$, Theorem 1 (a) reduces to the following corollary.

Corollary 4 ([14, Theorem 5 , page 724$])$. If $f \in \mathcal{S} \mathscr{T}(1 / 2)$, then

$$
\operatorname{Re}\left(\frac{z^{2} f^{\prime \prime}(z)}{f(z)}+\frac{z f^{\prime}(z)}{f(z)}\right)>0
$$

in $|z|<\rho=\sqrt{8 \sqrt{2}-11}=0.56$. The number $\rho$ is sharp.

Theorem 5. Let $f \in \mathcal{S} \mathscr{T}(1 / 2)$ and $\chi: \mathbb{D} \rightarrow \mathbb{C}$ be defined by

$$
\chi(z)=\frac{f(z)}{z}+f^{\prime}(z), \quad \chi_{i}(z)=\chi\left(\rho_{i} z\right), \quad i=1,2,3 .
$$

Then

(a) $\operatorname{Re} \chi_{1}(z)>\alpha, 0 \leq \alpha<1$, where $\rho_{1}$ is given by

$\rho_{1}= \begin{cases}\sqrt{\frac{7-8 \alpha}{5-8 \alpha+4 \sqrt{2} \sqrt{1-\alpha}},}, & 0 \leq \alpha<\frac{7+4 \sqrt{7}}{18}, \\ \frac{4-2 \alpha}{2 \alpha-1+\sqrt{1+4 \alpha}}, & \frac{7+4 \sqrt{7}}{18} \leq \alpha<1 .\end{cases}$

(b) $\left|\arg \chi_{2}(z)\right|<\gamma \pi / 2,0<\gamma \leq 1$, where $\rho_{2}=\rho_{2}(\gamma) \epsilon$ $(0,1)$ is the root of the equation

$$
\begin{aligned}
& \left(\sqrt{9+5 r^{2}\left(-2+r^{2}\right)}-2 r^{2}\right) \\
& \times \sqrt{16 r^{2}+\left(3+3 r^{2}-\sqrt{9+5 r^{2}\left(r^{2}-2\right)}\right)^{2}} \\
& +\left(\sqrt{9+5 r^{2}\left(r^{2}-2\right)}-11-11 r^{4}+5 r^{2}\right. \\
& \left.\quad \times\left(2+\sqrt{9+5 r^{2}\left(r^{2}-2\right)}\right)\right) \tan \left(\frac{\pi \gamma}{2}\right)=0 .
\end{aligned}
$$




\section{In particular,}

$$
\begin{gathered}
\rho_{2}\left(\frac{1}{4}\right) \simeq 0.257136, \quad \rho_{2}\left(\frac{1}{2}\right) \simeq 0.487998, \\
\rho_{2}\left(\frac{3}{4}\right) \simeq 0.674274, \quad \rho_{2}(1)=\sqrt{\frac{7}{5+4 \sqrt{2}}} \simeq 0.810465 .
\end{gathered}
$$

(c) Also $\left|\chi_{3}(z)-1\right|<\operatorname{Re} \chi_{3}(z)$, where $\rho_{3} \simeq 0.44915$ is given by the equation in $r$

$$
\begin{aligned}
& 24 r^{2} t_{0}-8+\left(-1+18 r^{2}-17 r^{4}\right) t_{0}^{2} \\
& \quad+2\left(1-r^{2}\right)^{2}\left(-1+3 r^{2}\right) t_{0}^{3}-\left(1-r^{2}\right)^{4} t_{0}^{4}=0
\end{aligned}
$$

and $t_{0}$ is given by the equation in $t$

$$
\begin{aligned}
& 24 r^{2}+2\left(-1+18 r^{2}-17 r^{4}\right) t \\
& \quad+6\left(1-r^{2}\right)^{2}\left(-1+3 r^{2}\right) t^{2}-4\left(1-r^{2}\right)^{4} t^{3}=0 .
\end{aligned}
$$

Each radius constant $\rho_{i}$ is sharp.

Proof. Let

$$
h(z)=\frac{1}{1-z}+\frac{1}{(1-z)^{2}} \quad(z \in \mathbb{D}) .
$$

Each $h_{i}(z)=h\left(\rho_{i} z\right), i=1,2,3$, is shown to, respectively, satisfy $\operatorname{Re} h_{1}(z)>\alpha,\left|\arg h_{2}(z)\right|<\gamma \pi / 2$, and $\left|h_{3}(z)-1\right|<$ $\operatorname{Re} h_{3}(z)$. Then, it follows from Lemma 2 that $\chi_{i}$ satisfies the required condition. (23),

(a) We claim that $\operatorname{Re} h(z)>\alpha$ in $|z|<\rho_{1}$. By (22) and

$$
\begin{aligned}
\operatorname{Re} h(z) & =R \cos \theta+R^{2} \cos 2 \theta \\
& =1+\frac{1}{2}\left(\left(1-3 r^{2}\right) t+\left(1-r^{2}\right)^{2} t^{2}\right) \quad\left(t:=R^{2}\right) \\
& :=\phi(t) .
\end{aligned}
$$

Case (i). Suppose $0 \leq \alpha<(7+4 \sqrt{7}) / 18$. In this case, it is shown that $\min \phi(t)>\alpha$ for $|z|<\rho_{1}$ over all $t$ in $(1 /(1+$ $\left.r)^{2}, 1 /(1-r)^{2}\right)$. Let $r<\rho_{1}$. It can be verified that

$$
\frac{\partial \phi(t)}{\partial t}=\frac{1}{2}\left(1-3 r^{2}+2\left(1-r^{2}\right)^{2} t\right)=0
$$

if $t=t_{0}=\left(3 r^{2}-1\right) /\left(2\left(1-r^{2}\right)^{2}\right), \partial^{2} \phi\left(t_{0}\right) / \partial t^{2}=\left(1-r^{2}\right)^{2}>0$, and that for $r \geq \sqrt{7}-2$,

$$
\frac{1}{(1+r)^{2}} \leq t_{0} \leq \frac{1}{(1-r)^{2}} .
$$

Thus for $\sqrt{7}-2 \leq r<\rho_{1}$,

$$
\min \phi(t)=\phi\left(t_{0}\right)=\frac{7-10 r^{2}-r^{4}}{8\left(1-r^{2}\right)^{2}}>\alpha .
$$

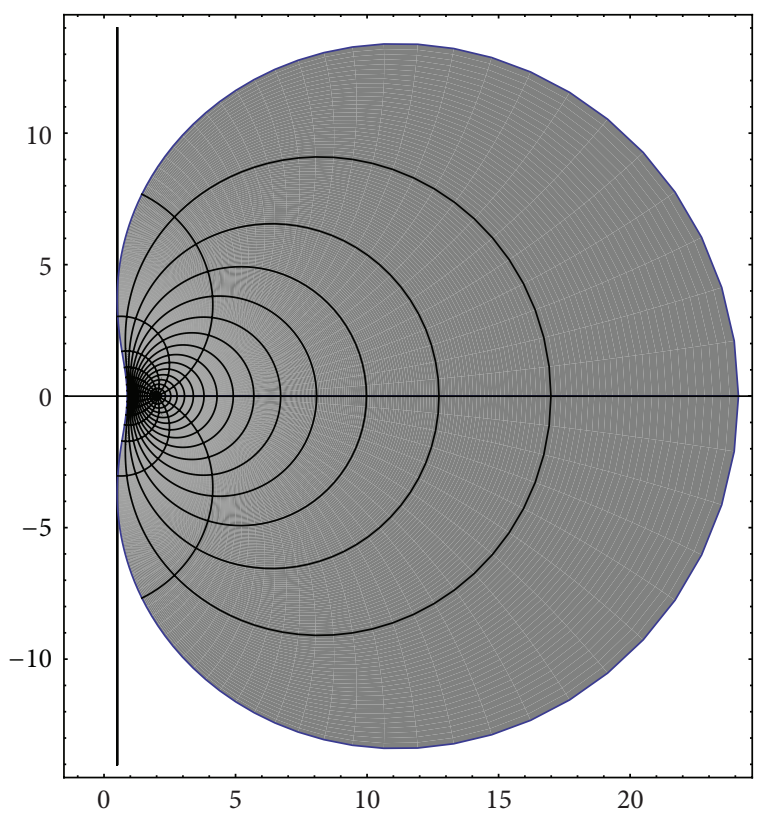

Figure 7: Image of $|z| \leq \sqrt{3 / 5}$ touches Re $w=0.5$.

On the other hand, if $r<\sqrt{7}-2$, then

$$
\min \phi(t)=\phi\left(\frac{1}{(1+r)^{2}}\right)=\frac{2+r}{(1+r)^{2}} .
$$

Since $g(r)=(2+r) /(1+r)^{2}$ is a decreasing function in $(0, \sqrt{7}-2)$,

$$
\begin{aligned}
\min \phi(t) & =\frac{(2+r)}{(1+r)^{2}}>\frac{2+\sqrt{7}-2}{(1+\sqrt{7}-2)^{2}} \\
& =\frac{\sqrt{7}}{(\sqrt{7}-1)^{2}}=\frac{4 \sqrt{7}+7}{18}>\alpha .
\end{aligned}
$$

Case (ii). For $(7+4 \sqrt{7}) / 18 \leq \alpha<1$, we prove that $\min \phi(t)>$ $\alpha$ in $|z|<\rho_{1}, t \in\left(1 /(1+r)^{2}, 1 /(1-r)^{2}\right)$. Let $r<\rho_{1}<\sqrt{7}-2$. As in Case (i), then

$$
\min \phi(t)=\phi\left(\frac{1}{(1+r)^{2}}\right)=\frac{(2+r)}{(1+r)^{2}}>\alpha .
$$

It is evident from the previous two cases that $\operatorname{Re} h_{1}(z)>$ $\alpha$ in $\mathbb{D}$. Figure 7 shows that, for $\alpha=0.5$, the radius $\rho_{1}=$ $\sqrt{3 / 5}$ is sharp.

(b) Let $h\left(r e^{i t}\right)=u+i v$. Then,

$$
\begin{gathered}
u=\frac{2\left(1+r^{2}+r^{2} \cos 2 t\right)-r\left(5+r^{2}\right) \cos t}{\left(1+r^{2}-2 r \cos t\right)^{2}}, \\
v=\frac{r\left(3+r^{2}-4 r \cos t\right) \sin t}{\left(1+r^{2}-2 r \cos t\right)^{2}} .
\end{gathered}
$$


By (67), it follows that

$$
\begin{aligned}
& \arg h\left(r e^{i t}\right) \\
& \quad=\arctan \left(\frac{r\left(3+r^{2}-4 r \cos t\right) \sin t}{2\left(1+r^{2}+r^{2} \cos 2 t\right)-r\left(5+r^{2}\right) \cos t}\right) .
\end{aligned}
$$

Let $g:[-1,1] \rightarrow \mathbb{R}$ be defined by

$$
g(x)=\frac{r\left(3+r^{2}-4 r x\right) \sqrt{1-x^{2}}}{2-r\left(5+r^{2}\right) x+4 r^{2} x^{2}} .
$$

(The case $-\sqrt{1-x^{2}}$ is similar.) A calculation shows that

$$
g^{\prime}(x)=\frac{r\left(1+r^{2}-2 r x\right)\left(r\left(7+r^{2}\right)-6 x\left(1+r^{2}\right)+4 r x^{2}\right)}{\sqrt{1-x^{2}}\left(2-r\left(5+r^{2}\right) x+4 r^{2} x^{2}\right)^{2}}
$$

Let

$$
x_{0}=\frac{3+3 r^{2}-\sqrt{9-10 r^{2}+5 r^{4}}}{4 r} \in(0,1]
$$

Then, $g^{\prime}\left(x_{0}\right)=0, g^{\prime}(x)>0$ for $x<x_{0}$, and $g^{\prime}(x)<$ 0 for $x>x_{0}$. Thus,

$$
g(x) \leq \max _{x \in[-1,1]} g(x)=g\left(x_{0}\right)
$$

Now (68) and (72) show that

$$
\left|\arg h\left(r e^{i t}\right)\right| \leq \frac{\gamma \pi}{2}
$$

provided

$$
\frac{r\left(3+r^{2}-4 r x_{0}\right) \sqrt{1-x_{0}^{2}}}{2-r\left(5+r^{2}\right) x_{0}+4 r^{2} x_{0}^{2}} \leq \tan \left(\frac{\gamma \pi}{2}\right)
$$

that is,

$$
\tan \left(\frac{\gamma \pi}{2}\right)-\frac{r\left(3+r^{2}-4 r x_{0}\right) \sqrt{1-x_{0}^{2}}}{2-r\left(5+r^{2}\right) x_{0}+4 r^{2} x_{0}^{2}} \geq 0
$$

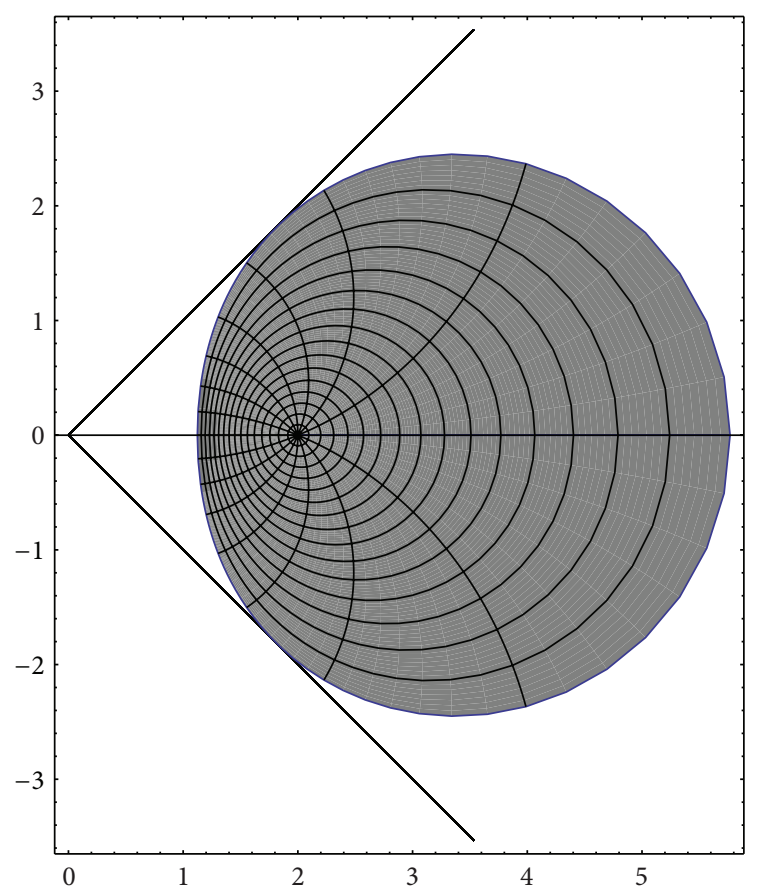

FIGURE 8: Image of $|z| \leq 0.487998$ touches $|\arg w|=\pi / 4$.

Thus, $\left|\arg h_{2}(z)\right|<\gamma \pi / 2$ in $\mathbb{D}$. Figure 8 shows that, for $\gamma=0.5$, the radius $\rho_{2}=0.487998$ is sharp.

(c) Proceeding similarly as in part (a),

$$
|h(z)-1|<\operatorname{Re} h(z)
$$

provided

$$
\begin{aligned}
& 24 r^{2} R^{2}-8+\left(18 r^{2}-1-17 r^{4}\right) R^{4} \\
& \quad-2\left(1-r^{2}\right)^{2}\left(1-3 r^{2}\right) R^{6}-\left(1-r^{2}\right)^{4} R^{8}<0 .
\end{aligned}
$$

Let $\phi:\left(1 /(1+r)^{2}, 1 /(1-r)^{2}\right) \rightarrow \mathbb{R}$ be defined by

$$
\begin{aligned}
\phi(t)= & 24 r^{2} t-8+\left(18 r^{2}-1-17 r^{4}\right) t^{2} \\
& -2\left(1-r^{2}\right)^{2}\left(1-3 r^{2}\right) t^{3}-\left(1-r^{2}\right)^{4} t^{4}
\end{aligned}
$$

Now

$$
\begin{aligned}
\phi^{\prime}(t)= & 24 r^{2}+2\left(18 r^{2}-1-17 r^{4}\right) t \\
& -6\left(1-r^{2}\right)^{2}\left(1-3 r^{2}\right) t^{2}-4\left(1-r^{2}\right)^{4} t^{3}
\end{aligned}
$$




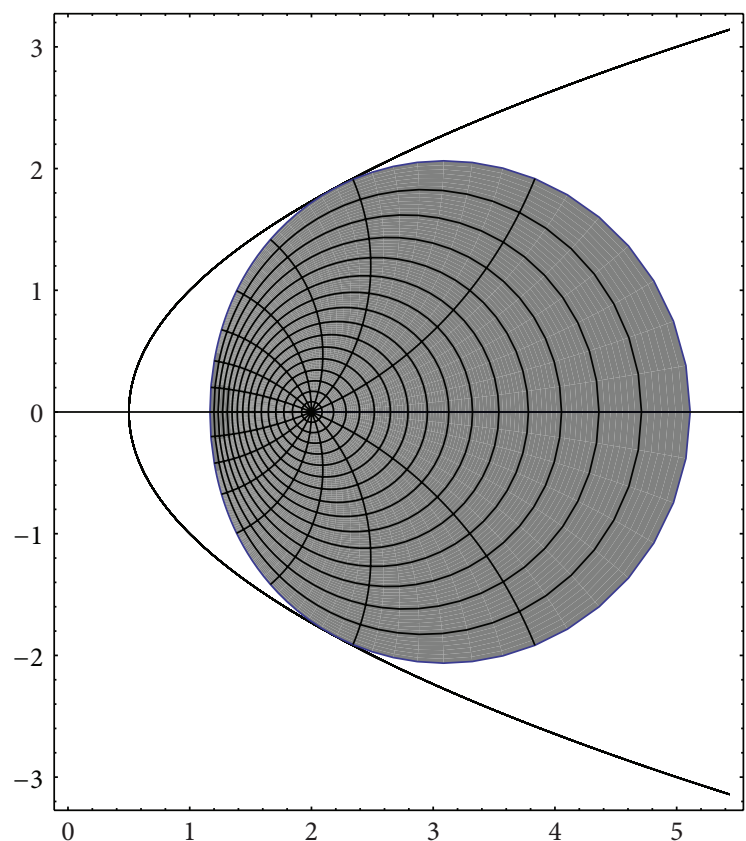

FIGURE 9: Image of $|z| \leq 0.44915$ touches $|w-1|=\operatorname{Re} w$.

Let $r<\rho_{3}$. Since

$$
\begin{aligned}
& \phi^{\prime}\left(\frac{1}{(1+r)^{2}}\right) \\
& \quad=\frac{4\left(-3+7 r+12 r^{2}+7 r^{3}+r^{4}\right)}{(1+r)^{2}}>0 \text { if } r>0.27606, \\
& \phi^{\prime}\left(\frac{1}{(1-r)^{2}}\right) \\
& \quad=\frac{4\left(-3-7 r+12 r^{2}-7 r^{3}+r^{4}\right)}{(1-r)^{2}}<0,
\end{aligned}
$$

there exists a unique $t_{0} \in\left(1 /(1+r)^{2}, 1 /(1-r)^{2}\right)$ such that $\phi^{\prime}\left(t_{0}\right)=0$ and $\max \phi(t)=\phi\left(t_{0}\right)$.

Then, for $0.27606<r<\rho_{3}$,

$$
\max \phi(t)=\phi\left(t_{0}\right)<0
$$

When $r \leq 0.27606$,

$$
\max \phi(t)=\phi\left(\frac{1}{(1+r)^{2}}\right)=\frac{4\left(-3+r^{2}\right)}{(1+r)^{2}}<0
$$

Evidently, $\phi(t)<0$ for $r<\rho_{3}$ and hence $\mid h_{3}(z)-$ $1 \mid<\operatorname{Re} h_{3}(z)$ in $\mathbb{D}$. Figure 9 shows that the radius $\rho_{3} \simeq$ 0.44915 is sharp.
Now, with $g(z)=z \in \mathcal{S} \mathscr{T}(1 / 2), i=1,2,3$,

$$
\begin{aligned}
& \frac{f(z) * z h_{i}(z)}{f(z) * z} \\
& =\frac{f(z) * z\left(1 /\left(1-\rho_{i} z\right)+1 /\left(1-\rho_{i} z\right)^{2}\right)}{f(z) * z} \\
& =\frac{f\left(\rho_{i} z\right)}{\rho_{i} z}+f^{\prime}\left(\rho_{i} z\right) \\
& =\chi\left(\rho_{i} z\right)=\chi_{i}(z) .
\end{aligned}
$$

Lemma 2, together with (83) and the corresponding inequality for the function $h_{i}$, shows that each function $\chi_{i}$ satisfies the required condition. For sharpness, consider the function $f_{0}(z)=z /(1-z) \in \mathscr{C} \mathscr{V} \subset \mathcal{S} \mathscr{T}(1 / 2)$. Clearly

$$
\frac{f_{0}(z)}{z}+f_{0}^{\prime}(z)=\frac{1}{1-z}+\frac{1}{(1-z)^{2}}=h(z) ;
$$

hence the fact that the number $\rho_{i}$ is sharp follows from the definition of $h$.

For $\alpha=0$, Theorem 5(a) reduces to the following corollary.

Corollary 6 ([14, Theorem 3 , page 722$])$. If $f \in \mathcal{S} \mathscr{T}(1 / 2)$, then

$$
\operatorname{Re}\left(\frac{f(z)}{z}+f^{\prime}(z)\right)>0
$$

in $|z|<\rho=\sqrt{4 \sqrt{2}-5} \simeq 0.81$. The number $\rho$ is sharp.

Theorem 7. Let $f \in \mathscr{C} \mathscr{V}, \chi: \mathbb{D} \rightarrow \mathbb{C}$ be defined by

$$
\begin{gathered}
\chi(z)=\left(1+\frac{z f^{\prime \prime}(z)}{f^{\prime}(z)}\right)+\frac{1}{f^{\prime}(z)} \\
\chi_{i}(z)=\chi\left(\rho_{i} z\right), \quad i=1,2 .
\end{gathered}
$$

Then,

(a) $\operatorname{Re} \chi_{1}(z)>\alpha, 0 \leq \alpha<1$, where $\rho_{1}=\sqrt{3-\sqrt{5+2 \alpha}}$;

(b) $\left|\arg \chi_{2}(z)\right|<\gamma \pi / 2,0<\gamma \leq 1$, where $\rho_{2}=\rho(\gamma) \epsilon$ $(0,1)$ is the root of the equation in $r$

$$
\begin{aligned}
& 2 r^{2} \sqrt{1-x_{0}^{2}}\left(r-\left(3+r^{2}\right) x_{0}+2 r x_{0}^{2}\right)+\tan \left(\frac{\pi \gamma}{2}\right) \\
& \quad \times\left(2-r^{2}-r^{4}-4 r x_{0}+\left(6 r^{2}+2 r^{4}\right) x_{0}^{2}-4 r^{3} x_{0}^{3}\right)=0,
\end{aligned}
$$

and $x_{0} \in[-1,1]$ is the root of the equation

$$
\begin{aligned}
6 & -5 r^{2}-4 r^{4}-r^{6}+\left(-6 r+15 r^{3}+7 r^{5}\right) x_{0} \\
& +\left(-12-8 r^{2}-16 r^{4}\right) x_{0}^{2}+\left(24 r+16 r^{3}\right) x_{0}^{3} \\
& -16 r^{2} x_{0}^{4}=0 .
\end{aligned}
$$


In particular,

$$
\rho_{2}\left(\frac{1}{2}\right) \simeq 0.6355, \quad \rho_{2}(1)=\sqrt{3-\sqrt{5}}
$$

The radii are sharp.

Proof. Let

$$
h(z)=\frac{2}{1-z}+(1-z)^{2}-1 \quad(z \in \mathbb{D}) .
$$

(a) We claim that $\operatorname{Re} h(z)>\alpha$ in $|z|<\rho_{1}$. By (22) and (23), it follows that

$$
\begin{aligned}
\operatorname{Re} h(z) & =2 R \cos \theta-1+\frac{\cos 2 \theta}{R^{2}} \\
& =\frac{1+t^{2}+r^{4} t^{2}+2 t^{3}-2 r^{2}\left(t+t^{2}+t^{3}\right)}{2 t^{2}} \quad\left(t:=R^{2}\right) \\
& :=\phi(t) .
\end{aligned}
$$

A calculation shows that $\phi^{\prime}(t)=0$ if $t=t_{0}=1 \in(1 /(1+$ $\left.r)^{2}, 1 /(1-r)^{2}\right), \phi^{\prime \prime}\left(t_{0}\right)=3-2 r^{2}>0$, and that

$$
\min \phi(t)=\phi(1)=\frac{1}{2}\left(4-6 r^{2}+r^{4}\right)>\alpha
$$

over all $t \in\left(1 /(1+r)^{2}, 1 /(1-r)^{2}\right)$ provided

$$
r^{4}-6 r^{2}+4-2 \alpha>0
$$

This inequality reduces to $r \leq \rho_{1}$. Thus, $\operatorname{Re} h_{1}(z)>$ $\alpha$ in $\mathbb{D}$. Figure 10 shows that, for $\alpha=0.5$, the radius $\rho_{1}=$ $\sqrt{3-\sqrt{6}}$ is sharp.

(b) Let $h\left(r e^{i t}\right)=u+i v$. Then

$$
\begin{aligned}
u= & \left(2(1-r \cos t)+\left(r^{2}\left(\cos ^{2} t-\sin ^{2} t\right)-2 r \cos t\right)\right. \\
& \left.\times\left(1+r^{2}-2 r \cos t\right)\right) \\
& \times\left(1+r^{2}-2 r \cos t\right)^{-1}, \\
v= & \frac{2 r \sin t\left(1-(1-r \cos t)\left(1+r^{2}-2 r \cos t\right)\right)}{1+r^{2}-2 r \cos t} .
\end{aligned}
$$

By (94), it follows that

$$
\begin{aligned}
\arg h\left(r e^{i t}\right) & \\
=\arctan ( & \left(2 r^{2}\left(-r+\cos t\left(3+r^{2}-2 r \cos t\right)\right) \sin t\right) \\
\times & \left(2+2 r^{2}-r\left(4+3 r^{2}\right) \cos t\right. \\
& \left.\left.+r^{2}\left(3+r^{2}\right) \cos 2 t-r^{3} \cos 3 t\right)^{-1}\right) .
\end{aligned}
$$

Let

$$
\begin{aligned}
g(x)= & \left(2 r^{2}\left(-r+x\left(3+r^{2}-2 r x\right)\right) \sqrt{1-x^{2}}\right) \\
& \times\left(2+2 r^{2}-r\left(4+3 r^{2}\right) x\right. \\
& \left.+r^{2}\left(3+r^{2}\right)\left(2 x^{2}-1\right)-r^{3}\left(4 x^{3}-3 x\right)\right)^{-1} .
\end{aligned}
$$

A calculation shows that there exists $x_{0} \in[0,1]$ such that $g^{\prime}\left(x_{0}\right)=0$ and $g^{\prime \prime}\left(x_{0}\right)<0$. Thus

$$
g(x) \leq g\left(x_{0}\right), \quad x \in[-1,1] .
$$

By (95), (96), and (97), evidently

$$
\left|\arg h\left(r e^{i t}\right)\right| \leq \frac{\gamma \pi}{2}
$$

if

$$
\begin{aligned}
& \frac{2 r^{2}\left(-r+x_{0}\left(3+r^{2}-2 r x_{0}\right)\right) \sqrt{1-x_{0}^{2}}}{2-r^{2}-r^{4}-4 r x_{0}+\left(6 r^{2}+2 r^{4}\right) x_{0}^{2}-4 r^{3} x_{0}^{3}} \\
& \quad \leq \tan \left(\frac{\gamma \pi}{2}\right) ;
\end{aligned}
$$

that is,

$$
\begin{aligned}
& 2 r^{2} \sqrt{1-x_{0}^{2}}\left(r-\left(3+r^{2}\right) x_{0}+2 r x_{0}^{2}\right) \\
& \quad+\tan \left(\frac{\gamma \pi}{2}\right)\left(2-r^{2}-r^{4}-4 r x_{0}+\left(6 r^{2}+2 r^{4}\right) x_{0}^{2}-4 r^{3} x_{0}^{3}\right) \\
& \quad \geq 0 .
\end{aligned}
$$

Thus, $\left|\arg h_{2}(z)\right|<\gamma \pi / 2$ in $\mathbb{D}$. Figure 11 shows that, for $\gamma=0.5$, the radius $\rho_{2} \simeq 0.6335$ is sharp. Then,

To conclude the proof, let $g(z)=z /\left(1-\rho_{i} z\right)^{2} \in \mathcal{S} \mathscr{T}$.

$$
\begin{aligned}
& \frac{f(z) *\left(z /\left(1-\rho_{i} z\right)^{2}\right) h_{i}(z)}{f(z) *\left(z /\left(1-\rho_{i} z\right)^{2}\right)} \\
& =\frac{f(z) *\left(z /\left(1-\rho_{i} z\right)^{2}\right)\left(2 /\left(1-\rho_{i} z\right)-1+\left(1-\rho_{i} z\right)^{2}\right)}{f(z) *\left(z /\left(1-\rho_{i} z\right)^{2}\right)} \\
& =\frac{f(z) *\left(2 z /\left(1-\rho_{i} z\right)^{3}+z-z /\left(1-\rho_{i} z\right)^{2}\right)}{f(z) *\left(z /\left(1-\rho_{i} z\right)\right)} \\
& =\left(1+\frac{\rho_{i} z f^{\prime \prime}\left(\rho_{i} z\right)}{f^{\prime}\left(\rho_{i} z\right)}\right)+\frac{1}{f^{\prime}\left(\rho_{i} z\right)} \\
& =\chi\left(\rho_{i} z\right)=\chi_{i}(z) .
\end{aligned}
$$

As in the earlier proofs, Lemma 2 together with (101) and the corresponding inequality for the function $h_{i}$ shows 


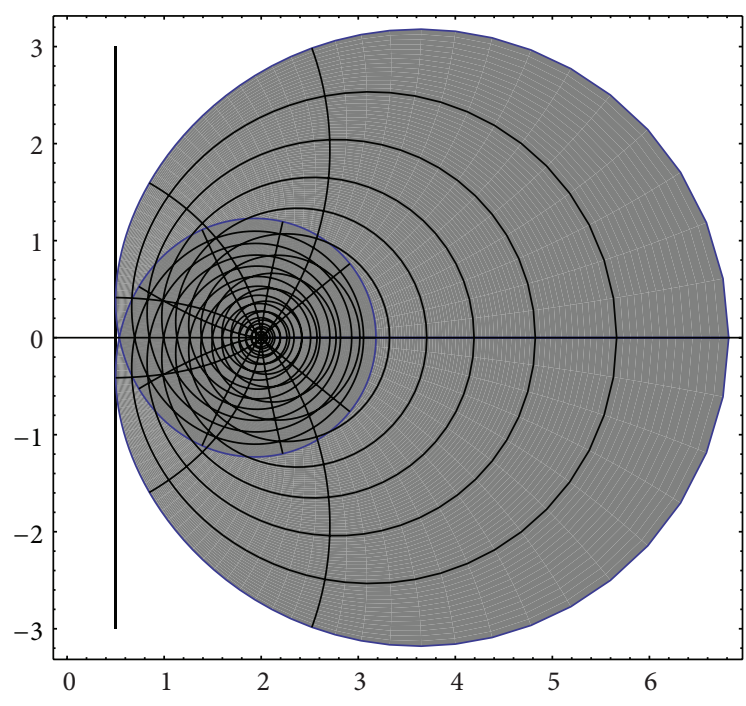

FIGURE 10: Image of $|z| \leq \sqrt{3-\sqrt{6}}$ touches Re $w=0.5$.

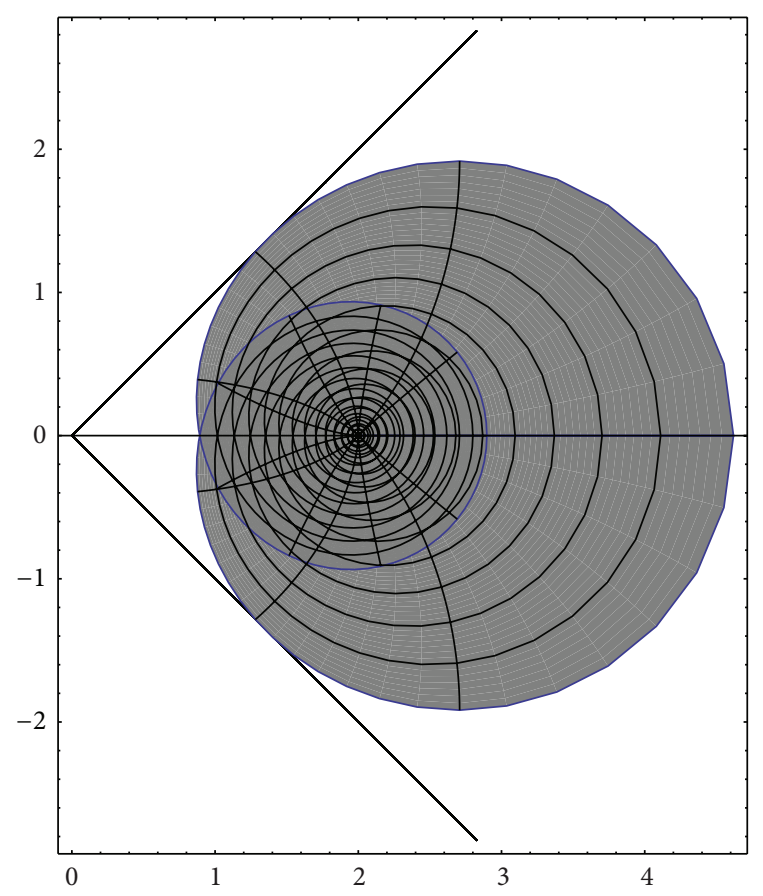

FIGURE 11: Image of $|z| \leq 0.6335$ touches $|\arg w|=\pi / 4$.

that the function $\chi_{i}$ satisfies the required condition. For sharpness, consider $f_{0}(z)=z /(1-z) \in \mathscr{C} \mathscr{V} \subset \mathcal{S} \mathscr{T}(1 / 2)$. Then,

$$
\left(1+\frac{z f_{0}^{\prime \prime}(z)}{f_{0}^{\prime}(z)}\right)+\frac{1}{f_{0}^{\prime}(z)}=\frac{2}{1-z}-1+(1-z)^{2}=h(z) .
$$

For $\alpha=0$, Theorem 7 (a) reduces to the following result.
Corollary 8 ([14, Theorem 4 , page 723$])$. If $f \in \mathscr{C} \mathscr{V}$, then

$$
\operatorname{Re}\left(\left(1+\frac{z f^{\prime \prime}(z)}{f^{\prime}(z)}\right)+\frac{1}{f^{\prime}(z)}\right)>0
$$

in $|z|<\rho=\sqrt{3-\sqrt{5}}=(\sqrt{5}-1) / \sqrt{2} \simeq 0.874032$. The result is sharp.

\section{Acknowledgments}

The work presented here is supported by a research university grant from Universiti Sains Malaysia, by a Senior Research Fellowship from the Council of Scientific and Industrial Research, New Delhi, and also by a grant from University of Delhi. The authors are thankful to the referee for the helpful comments.

\section{References}

[1] B. A. Uralegaddi, M. D. Ganigi, and S. M. Sarangi, "Univalent functions with positive coefficients," Tamkang Journal of Mathematics, vol. 25, no. 3, pp. 225-230, 1994.

[2] R. M. Ali, M. Haji Mohd, L. S. Keong, and V. Ravichandran, "Radii of starlikeness, parabolic starlikeness and strong starlikeness for Janowski starlike functions with complex parameters," Tamsui Oxford Journal of Information and Mathematical Sciences, vol. 27, no. 3, pp. 253-267, 2011.

[3] R. M. Ali, N. E. Cho, N. K. Jain, and V. Ravichandran, "Radii of starlikeness and convexity for functions with fixed second coefficient defined by subordination," Filomat, vol. 26, no. 3, pp. 553-561, 2012.

[4] S. Owa and H. M. Srivastava, "Some generalized convolution properties associated with certain subclasses of analytic functions," Journal of Inequalities in Pure and Applied Mathematics, vol. 3, no. 3, article 42, 13 pages, 2002.

[5] V. Ravichandran, M. Hussain Khan, H. Silverman, and K. G. Subramanian, "Radius problems for a class of analytic functions," Demonstratio Mathematica, vol. 39, no. 1, pp. 67-74, 2006.

[6] J. Sokół and J. Stankiewicz, "Radius of convexity of some subclasses of strongly starlike functions," Zeszyty Naukowe Politechniki Rzeszowskiej. Matematyka, no. 19, pp. 101-105, 1996.

[7] R. M. Ali, N. K. Jain, and V. Ravichandran, "Radii of starlikeness associated with the lemniscate of Bernoulli and the left-half plane," Applied Mathematics and Computation, vol. 218, no. 11, pp. 6557-6565, 2012.

[8] J. Sokół, "Coefficient estimates in a class of strongly starlike functions," Kyungpook Mathematical Journal, vol. 49, no. 2, pp. 349-353, 2009.

[9] J. Sokół, "Radius problems in the class $\mathcal{S} \mathscr{L}^{*}$," Applied Mathematics and Computation, vol. 214, no. 2, pp. 569-573, 2009.

[10] F. Rønning, "Uniformly convex functions and a corresponding class of starlike functions," Proceedings of the American Mathematical Society, vol. 118, no. 1, pp. 189-196, 1993.

[11] R. M. Ali and V. Ravichandran, "Uniformly convex and uniformly starlike functions," Mathematics Newsletter, vol. 21, no. 1, pp. 16-30, 2011.

[12] A. W. Goodman, Univalent Functions, vol. 1, Mariner, Tampa, Fla, USA, 1983. 
[13] P. L. Duren, Univalent Functions, vol. 259 of Grundlehren der Mathematischen Wissenschaften, Springer, New York, NY, USA, 1983.

[14] R. Singh and S. Paul, "Linear sums of certain analytic functions," Proceedings of the American Mathematical Society, vol. 99, no. 4, pp. 719-725, 1987.

[15] St. Ruscheweyh and T. Sheil-Small, "Hadamard products of Schlicht functions and the Pólya-Schoenberg conjecture," Commentarii Mathematici Helvetici, vol. 48, pp. 119-135, 1973. 


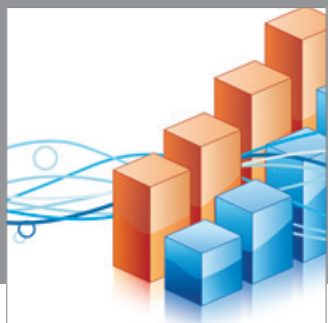

Advances in

Operations Research

mansans

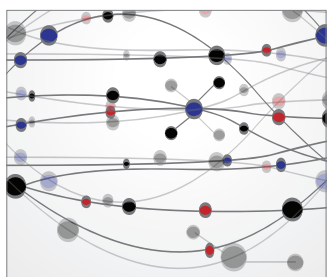

The Scientific World Journal
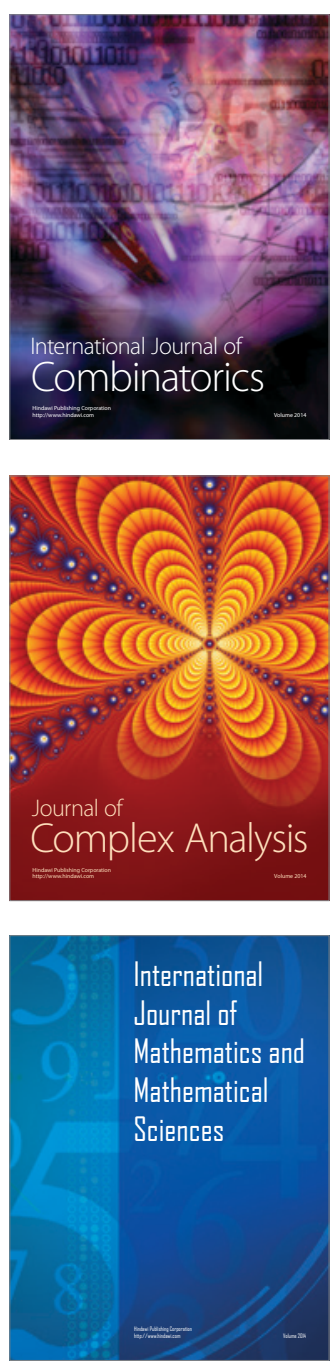
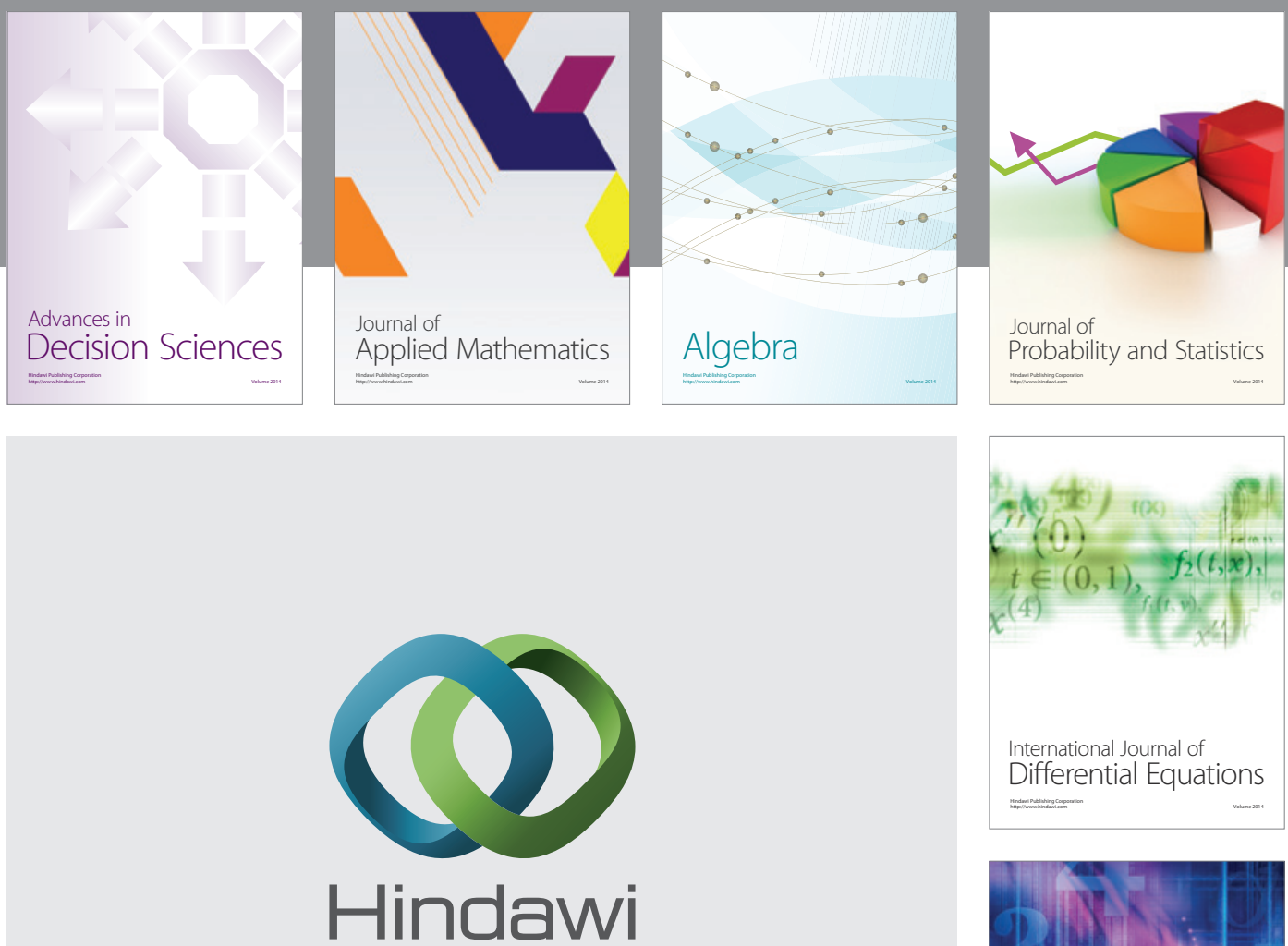

Submit your manuscripts at http://www.hindawi.com
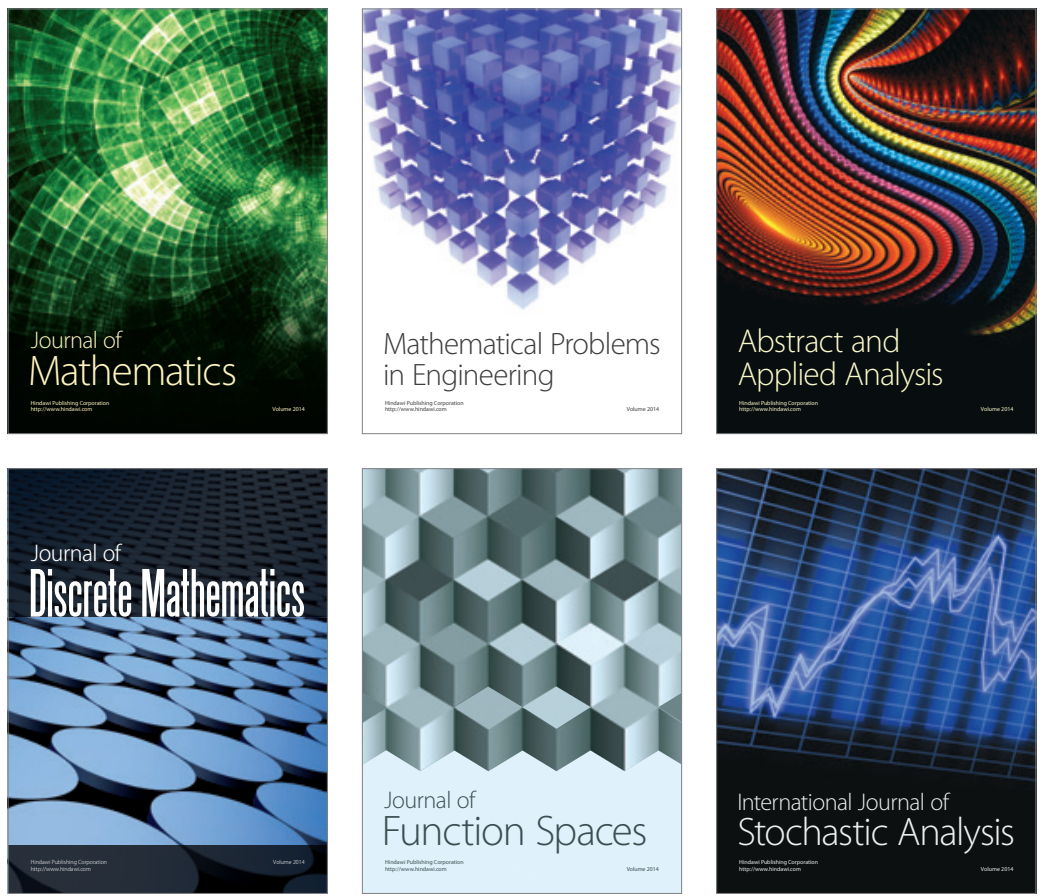

Journal of

Function Spaces

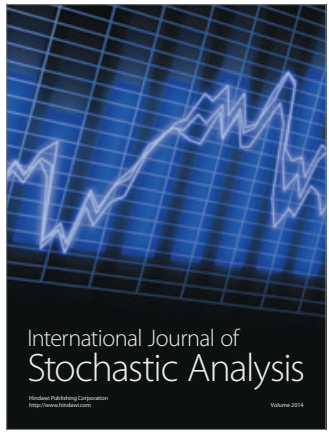

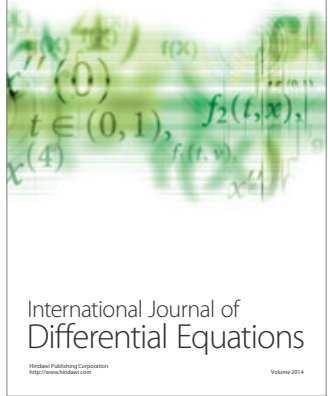
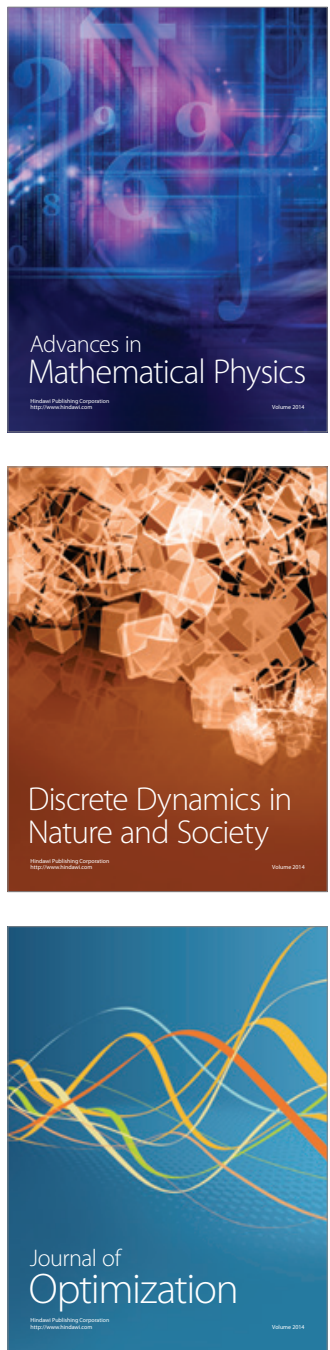